\title{
VERIFICAÇÃO DA INTERFERÊNCIA DO SOLVENTE PROPILENOGLICOL SOBRE O EFEITO DA PRÓPOLIS NA PRODUÇÃO DE LAGARTAS DE Bombyx mori L. (LEPIDOPTERA: BOMBICIDAE) COMPARADO AO EXTRATO ALCOÓLICO DE PRÓPOLIS
}

\author{
Jussara Ricardo Oliveira \\ Thomaz Mancini Carrenho Fabrin ${ }^{2}$ \\ Talita Aparecida Galbiati ${ }^{3}$ \\ Lorena Neri Barboza ${ }^{4}$ \\ Débora de Oliveira Furlanetto ${ }^{5}$ \\ Maria de Fátima Ferreira ${ }^{6}$ \\ Julio Cezar de Oliveira ${ }^{7}$ \\ Gustavo Ratti da Silva ${ }^{8}$ \\ Luciano Seraphim Gasques ${ }^{9}$
}

OLIVEIRA, J. R.; FABRIN, T. M. C.; GALBIATI, T. A.; BARBOZA, L. N.; FURLANETTO, D. de O.; FERREIRA, M. de F.; OLIVEIRA, J. C. de; SILVA, G. R. da; GASQUES, L. S. Verificação da interferência do solvente propilenoglicol sobre o efeito da própolis na produção de lagartas de Bombyx mori L. (Lepidoptera: Bombicidae) comparado ao extrato alcoólico de própolis. Arq. Ciênc. Vet.Zool. UNIPAR, Umuarama, v. 17, n. 1, p. 43-48, jan./mar. 2014.

RESUMO: Este experimento foi realizado no Laboratório de Sericicultura, no Campus Sede da Universidade Paranaense (UNIPAR) de Umuarama, no período de fevereiro a outubro de 2011, com o objetivo de verificar o efeito da própolis em diferentes dosagens na alimentação durante o desenvolvimento biológico do bicho-da-seda (Bombxy mori L.). O método empregado na parte experimental foi a pulverização do extrato glicólico de própolis, diluído em $500 \mathrm{~mL}$ de água destilada nas folhas de amoreira, nas seguintes dosagens, água-controle, $25 \mathrm{~mL}, 30 \mathrm{~mL}, 35 \mathrm{~mL}$ e $40 \mathrm{~mL}$ compondo os tratamentos: controle, $\mathrm{T}_{1}, \mathrm{~T}_{2}, \mathrm{~T}_{3}$ e $\mathrm{T}_{4}$ respectivamente. As folhas de amoreira foram fornecidas cinco vezes ao dia, durante o manejo alimentar. Verificou-se, pelos resultados obtidos, que as diferentes dosagens de própolis utilizadas não interferiram no ganho de peso das lagartas, no peso dos casulos verdes, no peso da casca sérica e crisálidas, quando comparado ao tratamento controle, mas quando se compara o Controle e T4 do ensaio da primavera, respectivamente, para os teores de seda bruto e líquido, há resultados significativos. Portanto, verificou-se que o extrato glicólico de própolis, em dosagens de $40 \mathrm{~mL}$, pode prejudicar o teor líquido de seda em uma produção de casulos, trazendo resultados pouco apreciados dentro da sericicultura.

PALAVRAS-CHAVE: Bicho-da-seda. Extrato glicólico de própolis. Sericicultura. Teor líquido de seda.

\section{INTERFERENCE OF PROPYLENEGLYCOL SOLVENT ON THE EFFECT OF PROPOLIS IN THE PRODUCTION OF SILKWORM Bombyx mori L. (LEPIDOPTERA: BOMBICIDAE) COMPARED TO PROPOLIS ALCOHOLIC EXTRACT}

\begin{abstract}
This experiment was performed at the Laboratory of Sericulture, at the main campus of University Paranaense (UNIPAR), in the city of Umuarama, from February to October 2011, in order to verify the effect of different doses of propolis in feeding during the biological development of silkworm (Bombyx mori L.). The method used in the experiment was the spraying of propolis glycolic extract dissolved in $500-\mathrm{mL}$ distilled water on the mulberry leaves in the following water-control dosages: $25 \mathrm{~mL}, 30 \mathrm{~mL}, 35 \mathrm{~mL}$ and $40 \mathrm{~mL}$ related to the treatment controls $\mathrm{T} 1, \mathrm{~T} 2, \mathrm{~T} 3$ and T4, respectively. Mulberry leaves were provided five times a day for feeding management. The results obtained showed that the different dosages of propolis used did not affect the weight gain of the larvae, the weight of green cocoons, shells and pupae when compared to the control treatment. However, when comparing the control and T4 from the Spring assay, respectively, to the levels of crude and net silk, significant results were noted. Thus, it can be concluded that propolis glycolic extract in 40-ml dosages may impair the net silk content in a cocoon production, presenting negative results in sericulture.
\end{abstract}

KEYWORDS: Silkworm. Glycolic extract of propolis. Sericulture. Silk net content.

DOI: https://doi.org/10.25110/arqvet.v17i1.2014.4916

${ }^{1}$ Centro Universitário de Maringá/UNICESUMAR. Endereço para correnpondência: Endereço: Av. Guedner, 1.610 - Jd. Aclimação, Maringá/PR. Email: jussara.ricardo.oliveira@gmail.com

${ }^{2}$ Universidade Estadual de Maringá/UEM - Programa de Pós-Graduação em Ecologia de Ambientes Aquáticos Continentais - Maringá/PR

${ }^{3}$ Universidade Paranaense/UNIPAR - Graduação em Ciências Biológicas - Umuarama/PR.

${ }^{4}$ Universidade Federal do Paraná/UFPR - Programa de Pós-Graduação em Farmacologia - Curitiba/PR.

${ }^{5}$ Universidade Paranaense/UNIPAR - Graduação em Biomedicina - Umuarama/PR.

${ }^{6}$ Universidade Paranaense/UNIPAR - Graduação em Ciências Biológicas - Umuarama/PR.

${ }^{7}$ Universidade Paranaense/UNIPAR - Graduação em Ciências Biológicas - Umuarama/PR

${ }^{8}$ Universidade Paranaense/UNIPAR - Graduação em Biomedicina - Umuarama/PR.

${ }^{9}$ Universidade Paranaense/UNIPAR - Professor do Curso de Ciências Biológicas - Umuarama/PR. 


\section{VERIFICACIÓN DE LA INTERFERENCIA DEL SOLVENTE PROPILENOGLICOL SOBRE EL EFECTO DE PROPÓLEOS EN LA PRODUCCIÓN DE GUSANO DE SEDA Bombyx mori L. (LEPIDOPTERA: BOMBICIDAE) COMPARADO AL EXTRACTO ALCOHÓLICO DE PROPÓLEOS}

RESUMEN: Este experimento se llevó a cabo en el Laboratorio de Sericultura, Campus Sede de la Universidad Paranaense (UNIPAR) de Umuarama, en el período de febrero a octubre de 2011, con el fin de verificar el efecto de propóleos en diferentes concentraciones en la alimentación durante el desarrollo biológico del gusano de seda (Bombyx mori L.). El método utilizado en el experimento fue la pulverización de extracto glicólico de propóleos, disuelto en 500 ml de agua destilada en las hojas de morera, en las siguientes dosis, agua control, $25 \mathrm{~mL}, 30 \mathrm{~mL}, 35 \mathrm{~mL}$, y $40 \mathrm{~mL}$ componiendo los tratamientos: control $\mathrm{T}_{1}, \mathrm{~T}_{2}, \mathrm{~T}_{3}$ e $\mathrm{T}_{4}$ respectivamente. A las hojas de morera se les han dado cinco veces al día, durante el manejo alimentar. Se verificó, por los resultados obtenidos, que las diferentes dosis de propóleos utilizados no afectaron en la ganancia de peso de los gusanos, en el peso de los capullos verdes, en el peso de la cáscara sérica y crisálidas, en comparación con el tratamiento de control, pero cuando se compara el Control y T4 del ensayo de la primavera, respectivamente, para los niveles de seda cruda y líquida, hay resultados significativos. Por lo tanto, se encontró que el extracto glicólico de propóleos, en dosis de 40 $\mathrm{mL}$, puede perjudicar la concentración líquida de seda en una producción de capullos, trayendo resultados poco apreciados dentro de la sericultura.

PALABRAS CLAVE: Gusano de seda. Extracto glicólico de propóleos. Sericultura. Contenido líquido de seda.

\section{Introdução}

A sericicultura é uma das atividades agroindustriais mais antigas que se tem registro na humanidade, datando provavelmente do período neolítico (PAPAVERO; PUJOL-LUZ, 2011). É também reconhecida por ser promissora e adequada ao desenvolvimento econômico e social do meio rural, principalmente em pequenas propriedades, proporcionando oportunidades de emprego com um investimento mínimo (SOUZA et al., 2010).

Sabbag, Nicodemo e Oliveira (2013) avaliaram a rentabilidade média da sericicultura em propriedades do estado de São Paulo e verificaram a possibilidade de lucros já no segundo ano da cadeia produtiva com Índice de Lucratividade superior a 50\% e Taxa Interna de Retorno de 9,68\%, entretanto esses valores são dependentes das cepas utilizadas no cultivo (ZANATTA et al. 2009).

O bicho-da-seda, sendo um inseto monofágico altamente especializado, consome e digere folhas de diferentes variedades de amoreira, mas o grau de utilização depende da digestibilidade e eficiência do alimento digerido que é convertido em substância corporal (SARKAR, 1993). Visando à completa nutrição da lagarta, não somente a variedade da amoreira é importante, como também o teor de nutrientes que ela possui, contribuindo, dessa forma, para um ótimo desenvolvimento e melhorando, consequentemente, a qualidade dos fios produzidos (MARCHI et al., 2009; MENEGUIM et al., 2010; PORTO, 2013). Das e Sikdar (1970); Tayade e Jawae (1984); Thanganmani e Vivekanandan (1984); Periasamy e Radhakrishan (1985); Polycarpo et al. (2012) comprovaram que as substâncias nutritivas diferem quantitativamente entre as variedades de amoreira e, por isso, têm significativa influência no desempenho biológico do bicho-da-seda e na produção de casulos.

Miranda, Takahashi e Silva (1998) verificaram que uma maneira de melhorar a qualidade nutricional dos alimentos é por meio da incorporação de nutrientes às folhas. A imersão de folhas de amoreira do cultivar Miura em soluções de ácido ascórbico nas concentrações de 0,5; 1,0 e $1,5 \%$ promoveram aumentos significativos no peso médio das lagartas, peso médio de pupa e peso médio de casulos, comparativamente aos outros tratamentos.

Em sericicultura, alguns autores encontraram que a administração oral de antibióticos, tais como a streptomicina e penicilina com a folha de amoreira, aumentaram o peso da lagarta do bicho-da-seda e o comprimento do fio da seda (MOORTHY et al., 1986). A própolis tem sido objeto de estudos farmacológicos, devido, por exemplo, às suas propriedades antibacteriana, antifúngica, antiviral, anti-inflamatória, hepatoprotetora, antioxidante, antitumoral e imunomodulatória. (BANKOVA, 2005; KOSALEC et al., 2005; ALENCAR et al., 2005; SIMÕES et al., 2008). Esse potencial biológico se deve a um sinergismo que ocorre entre seus muitos constituintes (MARCUCCI, 1996). Neste sentido, é importante para a sericicultura estudar a ação efetiva da própolis no desenvolvimento do bicho-da-seda.

Ao avaliar a eficiência do processo extrativo (alcoólico e propilenoglicol) de acordo com a concentração de própolis utilizando-se diversos líquidos extratores e, ao mesmo tempo, selecionar o extrato que apresente melhor atividade in vitro frente a uma coleção de leveduras isoladas de onicomicoses, Longhini et al. (2007) concluíram que entre os parâmetros estabelecidos houve a influência do glicol na seleção de substâncias extraídas.

O extrato de própolis obtido com glicóis propicia formas farmacêuticas emulsionadas mais estáveis, tornando-se importante o estudo de Franco e Bueno (1999) por possibilitar informações que assegurem o sucesso da substituição, com vantagens, do álcool 93,7 (p/p) como líquido extrator no preparo de extratos de própolis (LONGHINI et al., 2007).

O propilenoglicol é um solvente viscoso, utilizado em uma grande variedade de formulações farmacêuticas de uso tópico, devido a sua propriedade emoliente (DIEMUNSCH; MARTHIS, 1980). É também largamente utilizado na preparação de extratos vegetais, conhecidos como extratos glicólicos.

Como o extrato glicólico de própolis parece ser menos agressivo às lagartas do bicho-da-seda, o objetivo deste trabalho foi o de caracterizar a interferência do solvente propilenoglicol sobre o efeito da própolis na alimentação do Bombyx mori, verificando sua influência nos aspectos quantitativos e qualitativos da produção, principalmente na produção de casulos e no teor líquido de seda, que representam indicadores econômicos importantes para o sericicultor. 


\section{Material e Métodos}

Para o manejo de criação foram utilizadas lagartas híbridas comerciais, no início do $3^{\circ}$ ínstar, adquiridas da Indústria de Fiação Sedas Shoei Bratac S/A. O experimento foi realizado no Laboratório Experimental de Sericicultura, da Universidade Paranaense, Campus Sede - UNIPAR/PR, no município de Umuarama no período de fevereiro a outubro de 2011.

As folhas de amoreira utilizadas na alimentação foram da variedade Miura, cujos ramos e folhas eram colhidos nas horas mais frescas do dia sendo que, para melhor acondicionamento, as folhas eram armazenadas em sacos plásticos com o intuito de manter a turgescência delas.

Para a quantificação do desempenho biológico, foram contadas e separadas 40 lagartas por parcela, com cinco repetições para cada tratamento. Os parâmetros avaliados foram o peso inicial ( $4^{\circ}$ instar), peso final ( $5^{\circ}$ instar), peso das crisálidas, peso da casca sérica, teor de seda bruta e teor de seda líquida; para as avaliações foram utilizadas 30 lagartas, escolhidas de forma aleatória, das 40 que haviam por parcela.

As lagartas foram criadas em gavetas de criação de madeira (total de 25 gavetas), com dimensões de $0,90 \mathrm{~m}$ de comprimento, $0,60 \mathrm{~m}$ de largura e $0,10 \mathrm{~m}$ de altura com fundo tipo estrado, forradas com folhas de papel kraft, acondicionadas em uma estrutura de ferro de $1,75 \mathrm{~m}$ de altura $X 1,90 \mathrm{~m}$ de largura X $0,60 \mathrm{~m}$ de profundidade subdividida em três partes para comportar 12 gavetas, num total de 36 gavetas. As lagartas foram criadas nestas instalações do $4^{\circ}$ ao $5^{\circ}$ instar até a confecção dos casulos, após o $10^{\circ}$ dia da confecção, realizou-se o manejo de coleta para as análises dos parâmetros referentes aos casulos. O ambiente de criação e os materiais utilizados foram desinfetados com solução de formaldeído a $37 \%$ antes do início do experimento.

Para a alimentação das lagartas foram realizados cinco tratos diários ad libitum às 7h:30min, 10h:30min, 13h:30min, 16h:30min e 19h:30min, com folhas de amoreira previamente pulverizadas com diferentes dosagens do extrato glicólico de própolis diluídas em $500 \mathrm{~mL}$ de água destilada, que constituíram o Controle (somente água, sem adição de própolis) e quatro tratamentos: $\mathrm{T} 1=25 \mathrm{~mL}$ de própolis; $\mathrm{T} 2=$ $30 \mathrm{~mL}$ de própolis; $\mathrm{T} 3=35 \mathrm{~mL}$ de própolis; $\mathrm{T} 4=40 \mathrm{~mL}$ de própolis. O tratamento Controle teve como objetivo umedecer as folhas para se igualar ao manejo de pulverização com dosagens de própolis.

Um termo-higrômetro de máxima e mínima foi utilizado para a leitura da temperatura e umidade relativa do ar às $7 \mathrm{~h} 30 \mathrm{~min}$ e às $15 \mathrm{~h} 00 \mathrm{~min}$. Este procedimento foi adotado durante todo o período experimental, conforme descrito por Arnaut de Toledo et al. (1995). Para a confecção dos casulos foi utilizada uma cartela por parcela de um bosque giratório de papelão que ficaram sobre as lagartas.

Os teores bruto e líquido de seda, segundo Takahashi, Takahashi e Takahashi (2001), foram obtidos pesando-se 30 casulos completos (com crisálidas e espólio) para a obtenção do lote, cortam-se os 30 casulos e retiram-se a crisálida e o espólio, obtendo-se o peso dos casulos vazios, assim determinando a porcentagem de seda bruta pela fórmula: percentual de seda bruta $(\mathrm{SB})=$ peso da seda dos 30 casulos vazios (30 CV) dividido pelo peso de 30 casulos completos, cheios (30 CC) multiplicando-se por 100. O teor líquido de seda
(SL) é obtido descontando-se $24 \%$, que corresponde à parte que se perde durante a fiação.

$$
\begin{gathered}
S B=\frac{300 \mathrm{CV}}{30 \mathrm{CC}} \cdot 100 \\
S L=\text { SB. } 0,76
\end{gathered}
$$

A distribuição das parcelas foi totalmente aleatorizada, tendo um grupo controle com cinco repetições e quatro tratamentos com cinco repetições. Para as análises estatísticas foi utilizado o programa Statistica (STATSOFT, 2011), aplicando-se a análise de variância e teste de Tukey a nível de $5 \%$ de significância para comparação de médias.

\section{Resultados}

As condições de temperatura e umidade relativa do ar estiveram dentro do limite para criação do bicho-da-seda, uma média de $24,55^{\circ} \mathrm{C} \pm 1^{\circ} \mathrm{C}$ para a temperatura e $63,32 \% \pm$ $0,8 \%$ para a umidade relativa do ar, valores próximos quando comparados com Hanada e Watanabe (1986), em que comentam ser as condições ideais para a criação do bicho-da-seda, de $24^{\circ} \mathrm{C}$ de temperatura e $75 \%$ de umidade, não trazendo prejuízo à saúde das lagartas e para a qualidade dos casulos.

O peso inicial das lagartas de $4^{\circ}$ instar e peso final das lagartas de $5^{\circ}$ instar e os valores de ganho de peso das lagartas, por tratamento e por ensaio, estão apresentados nas Tabelas 1 e 2, e Figura 1, além disso, não houve diferenças significativas entre os tratamentos. Em ambos os ensaios o parâmetro ganho de peso não apresentou diferença significativa $(\mathrm{P}>0,05)$.

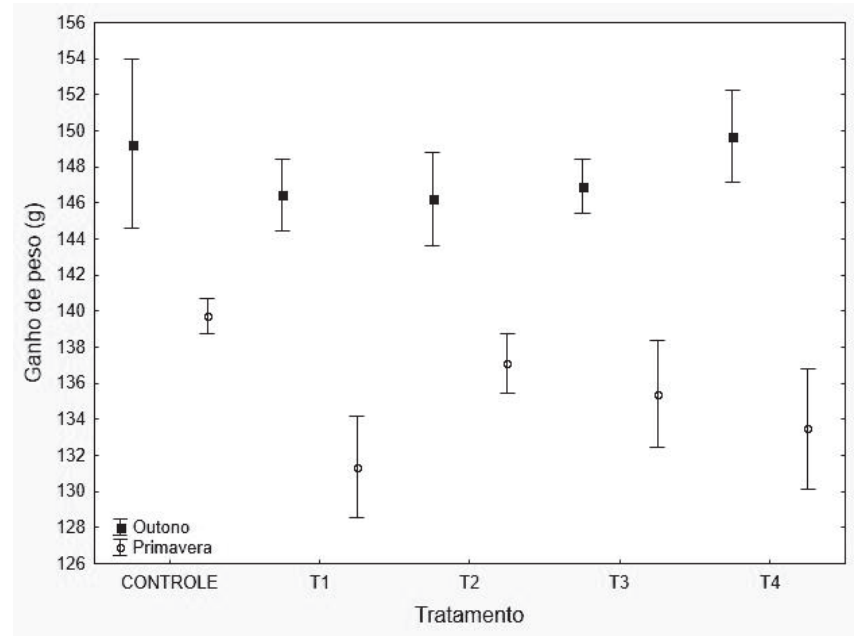

Figura 1: Ganho de peso (g) das lagartas em relação aos tratamentos. 
Tabela 1: Médias e desvios padrão dos pesos (g) inicial e final de 30 lagartas nos $4^{\circ}$ e $5^{\circ}$ instares, respectivamente e ganho de peso ( $\mathrm{g}$ ) das lagartas em relação aos tratamentos, referentes ao ensaio realizado durante outono de 2011.

\begin{tabular}{lccc}
\hline \multirow{2}{*}{$\begin{array}{c}\text { Trata- } \\
\text { mento }\end{array}$} & \multicolumn{3}{c}{ Ganho de peso $(\mathrm{g})$} \\
\cline { 2 - 4 } & $4^{\circ}$ instar & $5^{\circ}$ instar & $\begin{array}{c}\text { Ganho de peso } \\
(\mathrm{g})\end{array}$ \\
\hline $\begin{array}{l}\text { Contro- } \\
\text { le }\end{array}$ & $7,83 \pm 0,12$ & $157,09 \pm 10,56$ & $149,26 \pm 10,48$ \\
T1 & $7,97 \pm 0,46$ & $154,44 \pm 4,37$ & $146,47 \pm 4,43$ \\
T2 & $7,67 \pm 0,62$ & $153,90 \pm 6,39$ & $146,23 \pm 5,79$ \\
T3 & $7,53 \pm 0,43$ & $154,48 \pm 3,03$ & $146,95 \pm 3,36$ \\
T4 & $7,36 \pm 0,39$ & $157,07 \pm 5,59$ & $149,71 \pm 5,69$ \\
\hline
\end{tabular}

Tabela 2: Médias e desvios padrão dos pesos (g) inicial e final de 30 lagartas nos $4^{\circ}$ e $5^{\circ}$ instares, respectivamente e ganho de peso $(\mathrm{g})$ das lagartas em relação aos tratamentos, referentes ao ensaio realizado durante a primavera de 2011.

\begin{tabular}{lccc}
\hline \multirow{2}{*}{$\begin{array}{c}\text { Trata- } \\
\text { mento }\end{array}$} & \multicolumn{3}{c}{ Ganho de peso $(\mathrm{g})$} \\
\cline { 2 - 4 } & $4^{\mathrm{o}}$ ínstar & $5^{\mathrm{o}}$ instar & $\begin{array}{c}\text { Ganho de peso } \\
(\mathrm{g})\end{array}$ \\
\hline Contro- & & & $139,72 \pm 2,21$ \\
le & $5,50 \pm 0,09$ & $145,22 \pm 2,27$ & $131,37 \pm 6,33$ \\
T1 & $5,56 \pm 0,19$ & $136,93 \pm 6,48$ & $137,10 \pm 3,71$ \\
T2 & $5,72 \pm 0,25$ & $142,81 \pm 3,75$ & $130,6,69$ \\
T3 & $5,57 \pm 0,21$ & $140,99 \pm 6,77$ & $135,42 \pm 6,69$ \\
T4 & $5,62 \pm 0,24$ & $139,10 \pm 7,58$ & $133,48 \pm 7,50$ \\
\hline
\end{tabular}

Os percentuais do teor líquido de seda e teor bruto, dos ensaios realizados durante o outono e primavera estão representados na Figura 2 e 3, respectivamente, não havendo diferença significativa entre nenhum dos tratamentos durante o ensaio de outono, porém houve diferença significativa entre o Controle e T4 durante o ensaio da primavera $(\mathrm{P}<0,05)$, quanto ao teor de seda líquido.

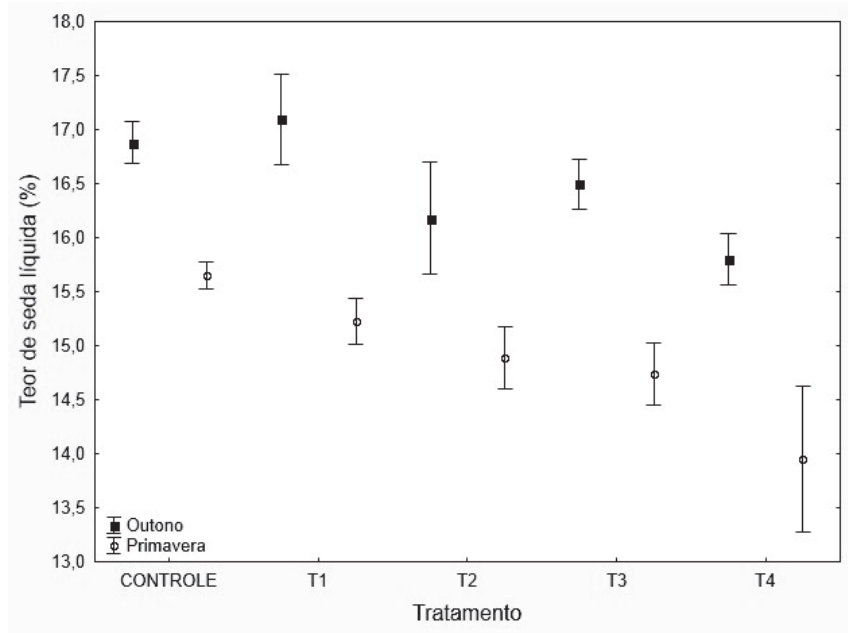

Figura 2: Teor de seda líquido (\%) em relação aos tratamentos.

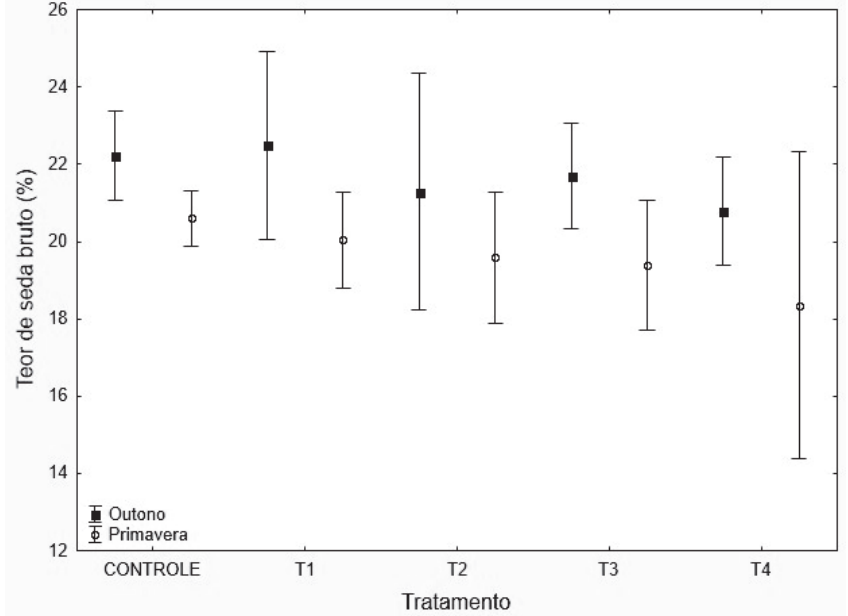

Figura 3: Teor de seda bruto (\%) em relação aos tratamentos.

\section{Discussão}

Quanto ao ganho de peso, segundo Fonseca e Fonseca (1988), a lagarta atinge seu maior peso um dia antes de parar de comer, e ao atingir sua plena "maturidade", está pronta para confeccionar o casulo. O T1 (primavera) apresentou uma tendência de um menor valor em relação aos demais, o mesmo acontecendo no experimento de Signorini et al. (2011) com a dosagem de $4 \mathrm{~mL}$ de extrato alcoólico de própolis, que pode ter ocorrido devido a redução da Eficiência da Conversão do Alimento Ingerido (ECI), que normalmente ocorre no último instar e este fato se deve a mudanças internas na fisiologia do inseto e a um dispêndio extra de energia na fase anterior à pupação, o que provoca um ganho de peso proporcionalmente menor pelo inseto nesta fase (SOO HOO; FRAENKEL, 1966).

No último dia do $5^{\circ}$ instar as glândulas sericígenas podem representar até $30 \%$ do peso corporal da lagarta, o que é fundamental para a confecção de um casulo com fios de qualidade. Segundo Porto (2000), o bicho-da-seda acumula reservas energéticas durante a fase larval para a produção de casulos e para as fases posteriores de seu ciclo biológico, assim o ganho de peso no final do $5^{\circ}$ instar é de grande importância.

Quando se compara o Controle com o T1 isto caracteriza um aumento de $0,084 \mathrm{~g}$ para o Controle. No último dia do $5^{\circ}$ instar as glândulas sericígenas podem representar até $30 \%$ do peso corporal da lagarta, justificando-se então esse ganho de peso.

O teor de seda bruta dos casulos provenientes do Controle e T4, ambos do ensaio realizado durante primavera, apresentaram $20,59 \%$ e $18,24 \%$, respectivamente, e esta é a parte que se utiliza para desenrolar o filamento (CIFUENTES; SOHN, 1998), enquanto os demais tratamentos apresentaram como valor 20,04\% (T1), 19,58\% (T2) e 19,37\% (T3). Embora esses valores não tenham apresentando diferenças significativas pode-se observar uma tendência negativa à medida que os valores das dosagens utilizadas foram aumentando.

Segundo Arnaut de Toledo et al. (1995), nem sempre existe relação entre o peso dos casulos e teor líquido de seda, assim como nem sempre o casulo mais pesado possui maior quantidade de seda, corroborando com os resultados 
deste trabalho. Normalmente, quanto menor o peso das crisálidas maior será o rendimento na produção de casulos (PORTO, 2005), o que foi ao encontro aos resultados obtidos nos quais ficou evidenciado entre o Controle (primavera) com teor líquido de seda de $15,66 \%$ que divergiu estatisticamente com o T4 (primavera), que teve o teor líquido de seda com valor de $13,95 \%$, em que o peso das crisálidas foi de $44,47 \mathrm{~g}$ (Controle) e 45,99g (T4).

Quando comparado o resultado com Signorini (2011), durante a primavera com dosagens de própolis diferentes, ou seja, T4 $=10 \mathrm{ml}$, verificou-se que o teor líquido de seda foi de $15,91 \%$, na dosagem máxima contra $15,66 \%$ para o tratamento controle do extrato glicólico de própolis. Estes dados parecem comprovar que o solvente alcoólico utilizado para a extração da própolis tem efeito tóxico para as lagartas, inclusive durante a formação de casulos (OLIVEIRA et al., 2010). Como a temperatura e a umidade relativa do ar são fatores limitantes dentro da sericicultura, acredita-se que, a diferença de $2,55^{\circ} \mathrm{C}$ talvez tenha influenciado também esses valores, porque afetou toda a parte experimental quando todos os dados dos parâmetros analisados foram bem menores.

A produção de casulos mais pesados e o teor de seda mais elevado são fatores que influenciam diretamente na rentabilidade da exploração, pois quanto mais pesado for o casulo, maior será a quantidade a ser comercializada e se o teor de seda também for elevado (acima de 17\%), maior será o preço do casulo, aumentando assim o lucro do sericicultor (TAKAHASHI; TAKAHASHI; TAKAHASHI, 2001).

\section{Conclusão}

A variável que apresenta maior importância econômica, o teor líquido de seda nos casulos obteve médias estatisticamente bem inferiores aos que normalmente é conseguido em uma criação comercial na dosagem de $40 \mathrm{~mL}$ de extrato glicólico de própolis, interferindo negativamente neste parâmetro.

O extrato glicólico de própolis cujo solvente utilizado na manipulação é o propilenoglicol, trouxe resultados positivos com valores bem mais elevados, quando comparado com o extrato alcoólico de própolis, podendo-se concluir que realmente certas substâncias são tóxicas para as lagartas podendo interferir na produção final.

A própolis na forma de extrato glicólico na dosagem acima de $35 \mathrm{~mL}$ pode ser prejudical para o desempenho biológico das lagartas de bicho-da-seda.

\section{Agradecimentos}

À Universidade Paranaense pelo incentivo para a realização do projeto de pesquisa e que o tornou possível pelo apoio financeiro e à BRATAC S/A por gentilmente ceder as lagartas utilizadas na parte experimental, sempre pronta a contribuir para o sucesso da exploração sericícola.

\section{Referências}

ALENCAR, S. M. et al. Composição química de Baccharis dracunculifolia. Ciência Rural, v. 35, p. 909-915, 2005.

ARNAUT DE TOLEDO, J. O. et al. Biological development of pure silkworm (Bombyx mori L.) breeds and hybrids in spring and fall. Sericologia, v. 35, n. 3, p. 563-567, 1995.

BANKOVA, V. Chemical diversity of propolis and the problem of standardization. Journal of Ethnopharmacology, v. 100, p. 114-117, 2005.

CIFUENTES, C. C. A.; SOHN, K. W. Manual técnico de sericicultura: cultivo de la morera y cria del gusano de seda em el trópico. Pereira: Convenio SENA-CDTS, 1998.

DAS, P. K.; SIKDAR, A. K. Evaluation of some improved strains of mulberry by feeding experiment. Indian Journal Sericulture, v. 9, p. 267-270, 1970.

DIEMUNSCH, A. M.; MARTHIS, C. Preparation et controle d'extraits végétaux ã usage cosmétologique. LaboPharma-Problëmes et Techiques, n. 294, p. 55-63, 1980.

FONSECA, A. S.; FONSECA, T. C. Cultura da amoreira e criação do bicho-da-seda. São Paulo: Nobel, 1988. 246 p.

FRANCO, S. I.; BUENO, J. H. F. Otimização do processo extrativo de própolis. Infarma, v.11, p. 48-51, 1999.

HANADA, Y.; WATANABE, J. K. Manual de criação do bicho-da-seda. Curitiba: Cocamar, 1986.

KOSALEC, I. et al. Flavonoid analysis and antimicrobial activity of commercially available propolis product. Acta Pharmacology, v. 55, p. 423-430, 2005.

LONGHINI, R. et al. Obtenção de extratos de própolis sob diferentes condições e avaliação de sua atividade antifúngica. Revista Brasileira de Farmacognosia, v. 17, n. 3, p. 388-395, 2007.

MARCHI, S. et al. Influência da alimentação na morfologia da glândula sericígena de lagartas no $5^{\circ}$ instar de Bombyx mori $\mathrm{L}$. utilizando diferentes cultivares de amoreira.

Arquivos de Ciências Veterinárias e Zoologia da Unipar, v.12, n.1, p. 17-22, 2009.

MARCUCCI, M. C. Propriedades biológicas e terapêuticas dos constituintes químicos da própolis. Química Nova, v.19, p. 529-536, 1996.

MENEGUIM, A. M. et al. Caracterização bromatológica de cultivares de amoreira, Morus spp., e determinação dos índices nutricionais de Bombyx mori L. (Lepidoptera: Bombycidae). Neotropical entomology, v. 39, n. 4, p. 506$512,2010$.

MIRANDA, J. E.; TAKAHASHI, R.; SILVA, A. F. Suplementação de ácido ascórbico à alimentação de Bombyx mori L. (Lepidoptera:Bombicidae). Revista de Agricultura, v. 73, n. 3, p. 367-372, 1998.

MOORTHY, C. V. N. et al. A review on the effects of 
different agents of nutrition on growth and economics of sericiculture. Sericologia, v. 26, n. 1, p. 35-42, 1986.

OLIVEIRA, J. R. et al. Avaliação da dosagem de própolis pulverizadas nas folhas de amoreira (Morus sp.) sobre o desenvolvimento biológico e produtivo do bicho-da-seda (Bombyx mori L.). Arquivos de Ciências Veterinárias e Zoologia da Unipar, v. 13, n. 2, p. 77-82, 2010.

PAPAVERO, N.; PUJOL-LUZ, J. R. Notas sobre o bicho-da-seda no folclore Chinês. Revista Brasileira de Entomologia, v. 55, n.1, p. 141-142, 2011.

PERIASAMY, K.; RADHAKRISHNAN, S. A quantitative study of food utilization and silk production in Bombyx mori $\mathrm{L}$. for evaluation of superior varieties of mulberry. Sericologia, La Mulatière, v. 24, n. 4, p. 491-502, 1985.

POLYCARPO, G. V. et al. Enriquecimento da dieta do bicho-da-seda com extrato hidrossolúvel de soja. Ciência Rural, Santa Maria, v. 42, n. 9, p.1669-1674, 2012.

PORTO, A. J. et al. Avaliação biológica do bicho-da-seda (Bombyx mori L.) alimentado com folhas de amoreira pulverizadas com extrato de Mirabilis jalapa. Arquivos do Instituto Biológico, v. 72, n. 4, p. 445-453, 2005.

PORTO, A. J. Efeito da idade de corte de dois cultivares de amoreira no desempenho e características do casulo do bicho-da-seda (Bombyx mori L.). 2000. 81 f. Dissertação (Mestrado em Zootecnia) - Faculdade de Medicina Veterinária e Zootecnia, Universidade Estadual Paulista, Botucatu, 2000.

PORTO, A. J. Suplementação da amoreira na alimentação do Bombyx mori L. Veterinária e Zootecnia, v. 20, n.1, p. 20-32, 2013.

SABBAG, O. J.; NICODEMO, D.; OLIVEIRA, J. E. M. Custos e viabilidade econômica da produção de casulos do bicho-da-seda. Pesquisa Agropecuária Tropical, v. 43, n. 2, p. 187-194, 2013.

SARKAR, A. Effects of feeding different races of silkworm (Bombyx mori) with mulberry (Morus indica L.) leafes varying in ployd level. Sericologia, v. 33, n. 1, p. 25-34, 1993.

SIGNORINI, T. et al. Efeito do própolis sobre o desenvolvimento do bicho-da-seda (Bombyx mori L.). Varia Scientia Agrárias, v. 2, n.1, p. 113-122, 2011.

SIMÕES, C. C.; ARAÚJO, D. B.; ARAÚJO, R. P. C. Estudo in vitro e ex vivo da ação de diferentes concentrações de extratos de própolis frente aos microrganismos presentes na saliva de humanos. Revista Braisileira de Farmacognosia, v. 18, p. 84-89, 2008.

SOO HOO, F. F.; FRAENKEL, G. The consumption digestion, and utilization of food plants by polyphagous insects, Prodenia eridania (Cramer). Journal of Insect
Physiology, v. 12, p. 711-730, 1966.

SOUZA, J. R. P. et al. Caracterização do grau tecnológico dos sericicultores no território Paraná Centro. Ciência Rural, v. 40, n.11, p. 2375-2380, 2010.

STATSOFT. (2011). STATISTICA (data analysis software system), version 10. www.statsoft.com.

TAKAHASHI, R.; TAKAHASHI, K. M.; TAKAHASHI, L. S. Sericicultura: uma promissora exploração agropecuária. Jaboticabal: FUNEP, 2001. 140 p.

TAYADE, D. S.; JAWAE, M. D. Studies on the comparative performance of silkworm races against different varieties of mulberry under Marathavada conditions. Sericologia, v. 24, n. 3, p. 361-364, 1984.

THANGAMANI, R.; VIVEKANANDAN, $\mathrm{M}$. Physiological studies and leaf nutrient analysis in the evaluation of best mulberry variety. Sericologia, v. 24, n. 3, p. 317-324, 1984.

ZANATTA, D. B. et al. Evaluation of economically important traits from sixteen parental strains of the silkworm Bombyx mori L (Lepidoptera: Bombycidae). Neotropical entomology, v. 38, n. 3, p. 327-331, 2009.

Recebido em: 10/05/2012 Aceito em: 02/05/2014 